\title{
ANGIOGRAPHY
}

\author{
By J. Norman Pattinson, M.B., B.Chir., D.M.R.D., F.F.R. \\ Assistant Radiologist, The Middlesex Hospital, London, W. I \\ Consultant Radiologist, The London Chest Hospital, London, E.2
}

Angiography in the investigation of cardiovascular disease consists of the radiological study of the heart and great vessels following the injection of a radio opaque solution. Two methods are in common use, Angiocardiography and Thoracic Aortography.

\section{Contrast Media}

In recent years, Diodone 70 per cent., NeoIopax 75 per cent., and Sodium Acetrizoate 70 per cent., have been used. Dotter et al. (1953), considered that the latter was the best for angiocardiography. These compounds are toxic and their rapid injection usually produces an intense sensation of heat and weakness and on occasion, nausea, vomiting and headache. More serious sequelae such as respiratory arrest, collapse, or death are infrequent. In the survey conducted by Dotter and Jackson (1950), 26 deaths were recorded in 6,824 angiocardiograms, a mortality of 0.38 per cent. Cyanotic congenital heart disease was present in 23 of the 26 patients. Death occurred following the second injection of contrast medium in nine patients and after the third injection in three others.

\section{Apparatus}

Much useful information can be obtained by the use of inexpensive single plane film changers, giving an exposure rate of one or two films per second. When films are exposed at such infrequent intervals however, errors in the interpretation of the shape of the cardiac chambers and the course of the circulation may occur. In addition transitory shunts may be missed and the true size of stenotic segments is difficult to evaluate.

Apparatus is now available which provides synchronous radiography in two projections at right angles to each other. The exposure rate can be varied up to 12 films per second. Lind and Wegelius (1949 and 1953b) have described the advantages of rapid biplane radiography.

Cine radiography is an alternative to 'direct' radiography (Janker, 1950 and $195 \mathrm{I}$ ). The previous disadvantages of high dosage of X-rays and poor film detail in comparison with ' direct, radiography, appears to have been largely over- $\vec{\omega}$ come by the development of the image amplifier. However, at present the latter allows only a field of 5 in. in diameter to be covered, and biplane models remain to be developed.

\section{Techniques}

Angiocardiography. The injection is made through a large bore cannula ( 1.5 to $2.5 \mathrm{~mm}$.) 은 inserted into an antecubital vein. We prefer to cut down and tie in the cannula using adequate TI local anaesthesia in all cases to avoid venospasm. It is desirable to inject all the contrast medium within two seconds. The use of a pressure injector and all metal syringe is advisable fo $\overrightarrow{0}$ amounts over $25 \mathrm{ml}$., and in all cases where catheter is used.

In the alternative method of ' selective' angio' cardiography advocated by Jonsson et al. (I949, I95 Ia and I953) the contrast medium is injected through the cardiac catheter after cardiac catheterization. This allows all the contrast medium to be delivered undiluted at the site under investigation. Great care must be taken to position the catheter correctly, or the injection may prove fatal.

Thoracic Aortography. One of the most widely used methods is catheterization of the aorta via the right radial artery (Jonsson et al., 195I Ib). The catheter is inserted until its tip lies halfway down the ascending aorta. If placed too high the catheter may recoil into the innominate artery during injection, or if placed too low the tip will be near the coronary ostia. In either of these events, the injection may be fatal due to the flow of highly concentrated contrast medium into these vessels.

Percutaneous insertion of a metal cannula into the aorta through the right common carotid artery was employed for a time by Jonsson. Sutton improved this technique by using a polythene tube.

Retrograde injection into the left common carotid (Burford and Carson, 1948) or left brachial arteries (Castellanos and Pereiras, 1939) has been used, and direct puncture of the ascending aorta 
(Nuvoli, 1936) or the aortic arch (Wickbom, 1952) has also been employed. Some of these latter techniques are more risky than the catheter methods.

No mortality statistics comparable with those for angiocardiography have been published. The risk of reaction to the contrast medium may be somewhat greater since its concentration in the aorta and its branches will usually be higher than in angiocardiography. There is also an additional risk due to technical errors in the positioning of the catheter.

\section{Dosage of Contrast Medium}

We now use between I to $1.2 \mathrm{ml}$., of contrast medium per kilo of body weight up to a total of about $65 \mathrm{ml}$., in an adult.

\section{Anaesthesia}

General anaesthesia (Cope, I953), is preferred for all patients under 16 years of age.

\section{General Indications}

Angiocardiography is of great value in cyanotic congenital heart disease where the right to left shunt can be well demonstrated. The anatomical defect may also be shown. It is less successful in outlining the abnormal communications in acyanotic cases with a left to right shunt. It is also useful for determining the site of stenosis in such conditions as pulmonary stenosis, subaortic stenosis and coarctation of the aorta. In acquired disease it is used in cases of aortic aneurysm, and also occasionally in cases of mitral valve disease.

Several abnormalities e.g. coarctation of the aorta, congenital anomalies of the aortic arch and its branches, patent ductus arteriosus and aortic aneurysms are better shown by Thoracic Aortography.

\section{Congenital Heart Disease (including the great vessels)}

Tetralogy of Fallot. Angiocardiography is of great value in this condition and some authorities (Dotter and Steinberg, I95 I) advocate its use in all cases before operation. It is required for diagnosis in only a small porportion of cases but may be important in planning the surgical approach e.g. systemic-pulmonary anastomosis or a direct operation on the stenosis.

The diagnostic features are the demonstration of an overriding aorta and the presence of infundibular or valvular stenosis. Opacification of the aorta occurs early as it fills from the right ventricle. The pulmonary stenosis is usually infundibular. Sometimes it is associated with valvular stenosis (Fig. I). In such cases the valves form a dome shaped filling defect and an infundibular chamber

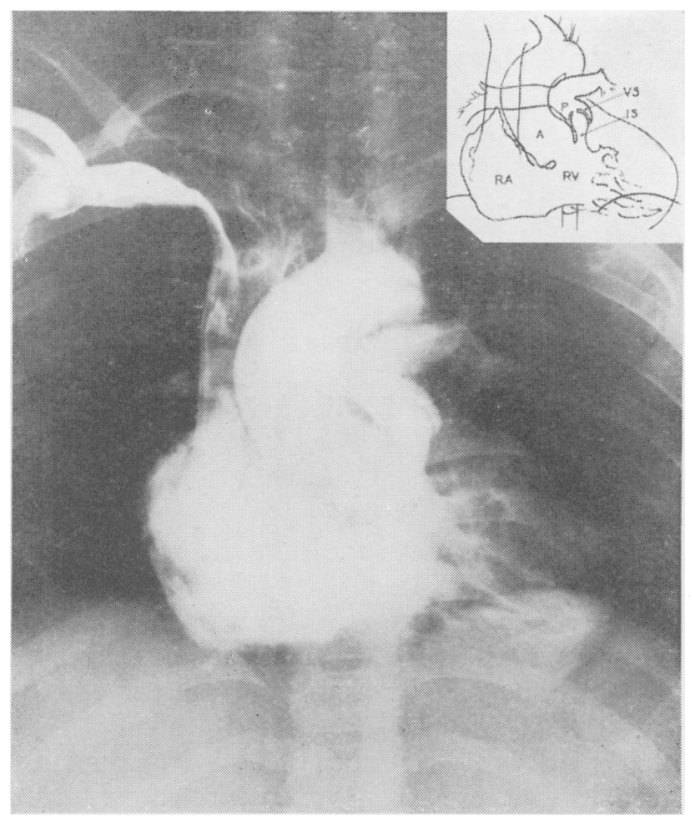

FIg. 1.-Tetralogy of Fallot. A.P. film, 1.75 sec Infundibular (I.S.) and valvular stenosis (P.S.).

may be seen between the two stenotic areas. Rarely the stenosis is purely valvular. The pulmonary trunk is usually small and may be atretic. It may, however, show some dilatation in the presence of valvular stenosis. The size of the right and left pulmonary arteries, which is difficult to evaluate on plain radiography is generally well shown and this may be of great importance to the surgeon. Rarely the left pulmonary artery is completely absent (Emanuel and Pattinson).

Other features which may be seen are enlargement of the superior vena cava, the right atrium, its appendix, and the right ventricle. Opacification of the pulmonary vessels is always diminished. It is very poor in pulmonary atresia, in which condition enlarged bronchial arteries may be visible passing down from the aorta to the hila. Some indication of the degree of override may be obtained from the size and density of the ascending aorta. With extreme override, the ascending aorta is greatly enlarged and becomes very dense, whereas its early opacification can be difficult to detect when the override is small. Anomalies of the aortic arch and its branches may be seen but will usually have been demonstrated previously at fluoroscopy. Most of the findings described above can be shown in the antero-posterior (A.P.) view. The pulmonary stenosis however, is often inadequately shown in this position, unless rapid serial 
radiography is employed and is best demonstrated in the right anterior oblique (R.A.O.) view.

The coexistence of a patent foramen ovale or atrial septal defect is not uncommon (pentalogy of Fallot). The presence of a right to left shunt through this septal defect can be detected in the A.P. view by the characteristic shape of the uppe: part of the left atrium. The size of this defect however, and the shunt through it (or through the ventricular septal defect) is best seen in the left anterior oblique (L.A.O.) position. This view is also the best to show the degree of dextro position of the aortic root.

\section{Persistent Truncus Arteriosus}

In this uncommon condition the aorta and the pulmonary artery are replaced by a single large arterial trunk, which arises from both ventricles. The truncus fills with contrast medium from the right ventricle and its ascending portion may be seen to give origin to a short pulmonary trunk which divides into right or left pulmonary arteries. Alternatively these arteries may arise directly from the truncus. In other cases the pulmonary arteries are absent and the lungs are supplied by large bronchial arteries; the condition is then indistinguishable by angiocardiography from pulmonary atresia (pseudo truncus arteriosus). Differentiation of extreme tetralogy of Fallot from truncus arteriosus may, however, be possible by the demonstration of a small pulmonary trunk arising from the right ventricle.

\section{Eisenmenger Complex}

The findings in many respects resemble those in tetralogy of Fallot from which anomaly it must be distinguished.

Angiocardiograms show enlargement of the right side of the heart and overriding of the aorta. The significant feature is the absence of any infundibular or valvular stenosis. The main pulmonary artery and its proximal branches are usually much dilated and may show prolonged retention of contrast medium due to the increased peripheral resistance.

Cardiac catheterization is advisable to demonstrate the absence of a systolic gradient between the right ventricle and pulmonary artery.

\section{Transposition of the Great Vessels}

These cyanotic lesions usually have multiple intracardiac defects with complex shunts which may not be fully elucidated during life even after angiocardiography and cardiac catheterization. In complete transposition the aorta arises entirely from the right ventricle and the pulmonary artery from the left ventricle; intercommunication between the pulmonary and systemic circulations

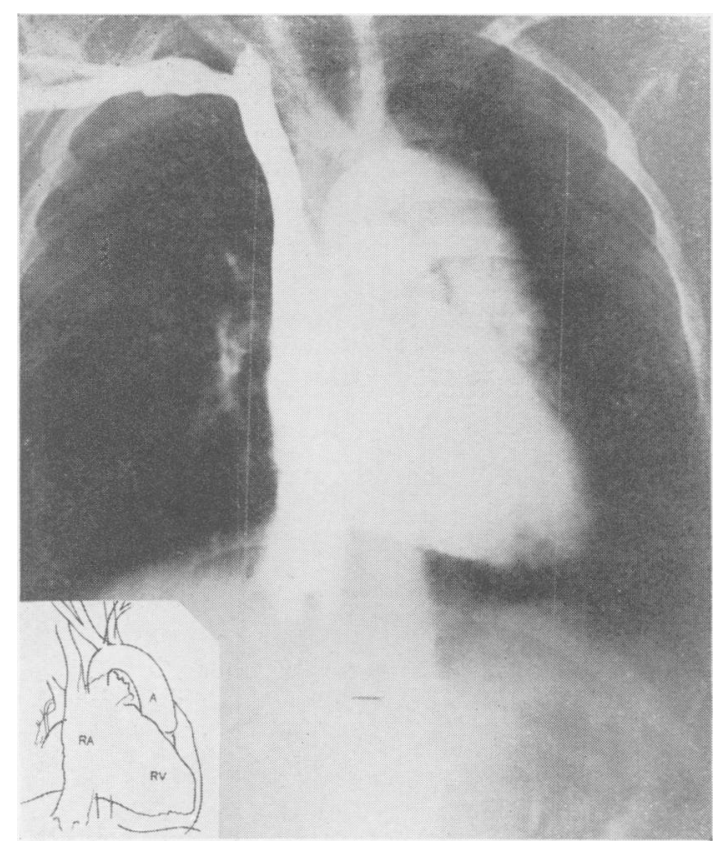

FIG. 2.-Transposition of the great vessels. A.P. film, I.5 sec. Aorta (A) arising anteriorly and to the left from the right ventricle. (Type 4, Castellanos et al., 1950.)

is necessary for survival. Most commonly this is an interatrial or interventricular septal defect of both, and sometimes a patent ductus arteriosus. Pulmonary stenosis may also be present. In incomplete transposition the pulmonary artery may arise from the right ventricle or override the interventricular septum.

Angiocardiograms show enlargement of the right atrium and ventricle with early opacification of the aorta as in the tetralogy of Fallot. The aorta rapidly becomes very dense, because it arises solely from the right ventricle but its ascending portion is not dilated as in tetralogy of Fallot or in truncus arteriosus (Fig. 2). The position of the aorta varies and Castellanos et al. (I950) have described four types. In all cases the lateral and L.A.O. views show that the aortic root arises very anteriorly and that the arch and ascending aorta form a wide arc. Usually little or no opacification of the left atrium, left ventricle and pulmonary vessels occurs since the small amount of contrast medium passing through the septal defect is rapidly diluted by the blood in the left side of the heart. In partial transposition better filling of the pulmonary vessels occurs.

\section{Tricuspid Atresia}

The fundamental defect is a non-functioning right ventricle. All the blood from the right 


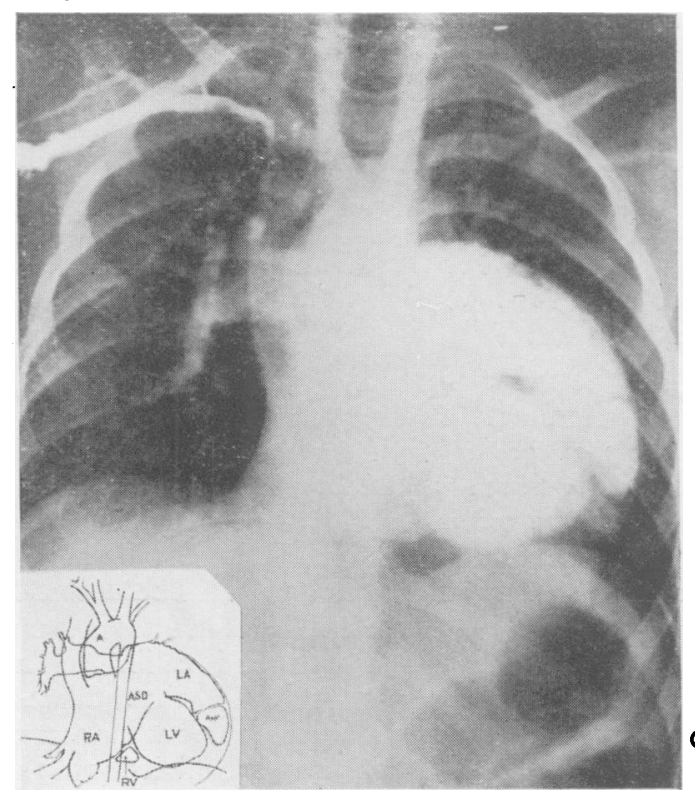

FIG. 3.-Tricuspid atresia. A.P. film, 3.3 sec. Large atrial septal defect (A.S.D.). Diminutive right ventricle (R.V.).

atrium passes through an atrial septal defect into the left atrium and left ventricle, both of which are grossly enlarged. The pulmonary artery may be supplied by the left ventricle through a ventricular septal defect and the hypoplastic right ventricle. If the pulmonary artery is atretic, the lungs are supplied by bronchial arteries or a patent ductus arteriosus. In either case the pulmonary blood flow is diminished. In many cases, there is partial or complete transposition of the great vessels and dextrocardia without situs inversus abdominalis is not uncommon.

The course of the circulation can frequently be demonstrated by angiocardiography. Attention should be paid to the size of the right atrium which is enlarged if the atrial septal defect is small. The right ventricle when filled, forms a small triangular or rounded opacity in the A.P. view, situated between the right atrium and left ventricle (Fig. 3). Some times the right ventricle fails to fill and a thick muscular wall is visible anterior to the left ventricle in the L.A.O. position. The aorta fills densely and rapidly unless transposed, while the pulmonary artery fills poorly unless it arises from the left ventricle.

\section{Simple Pulmonary Stenosis}

This condition has been discussed by Abrahams and Wood (195I). These cases (where the aorta arises normally from the left ventricle) may be divided into three groups:

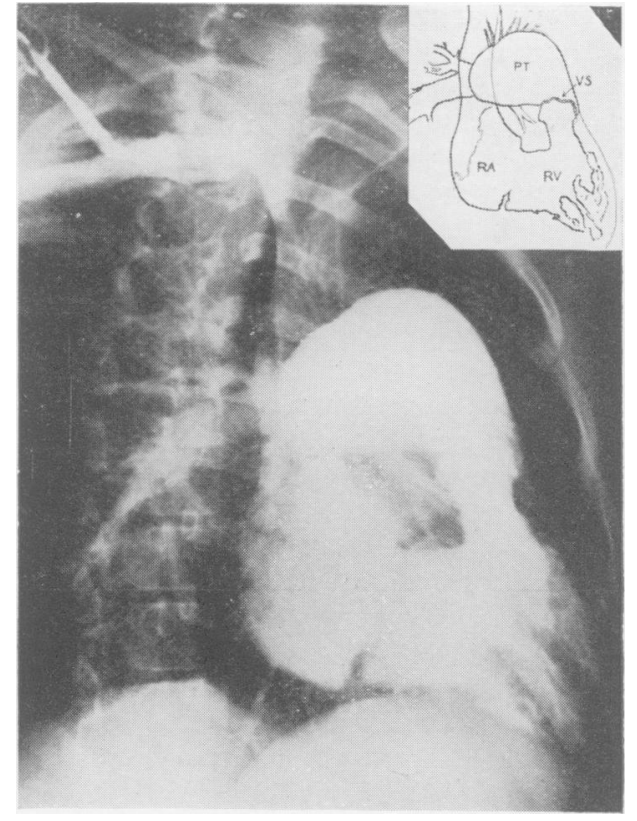

FIG. 4.-Simple pulmonary stenosis. R.A.O. film, 2 sec. Valvular stenosis (V.S.) with dilated pulmonary trunk (P.T.).

I. Isolated pulmonary stenosis; valvular orơ infundibular.

2. Valvular or infundibular stenosis with left to right shunt through an atrrial septal defect, ventricular septal defect or patent ductus arteriosus.

3. Valvular or infundibular stenosis with right to left shunt through an atrial or ventricular septal defect.

Angiocardiography is of value in all cases when surgery is contemplated, especially in the cyanotic cases of group 3, which may simulate the tetralogy of Fallot. The right atrium and ventricle are enlarged, particularly in the presence of an intracardiac shunt and their filling is prolonged. In the majority, the stenosis is located at the pulmonary valve. The fused leaflets produce a cone shaped filling defect bulging up into the pulmonary trunk (Fig. 4). A jet of contrast medium may be seen passing through the small central orifice of the valve. Great dilatation of the pulmonary trunk is usually present, except in cases where the stenosis is infundibular. The dilatation may also involve the right and left pulmonary arteries. Due to the stenosis, filling of the left atrium and ventricle is delayed and poor. The aorta is small in calibre and fills solely from the left ventricle.

Demonstration of the septal defect in cases of group 2 with a left to right shunt is difficult by 
intravenous angiocardiography. Indirect signs such as refilling of right atrium and ventricle from left side of the heart may be seen on later films, but they are not pathognomonic and must be evaluated with great caution. In cyanosed patients of group 3 (trilogy of Fallot) the pulmonary stenosis and right to left shunt can be well shown by angiocardiography. The shunt is best demonstrated in the L.A.O. position. The rapid transit of contrast medium through the left atrium and ventricle into the aorta may simulate the appearances seen in tetralogy or pentalogy of Fallot if films are exposed at infrequent intervals.

Differentiation between these conditions is important for anastomotic operations are inadvisable in simple pulmonary stenosis; valvotomy or infundibular resection is the operation of value.

\section{Atrial Septal Defect}

As a rule, intravenous angiocardiography is of little help in this condition due to the predominant left to the right shunt. In infants, however, Lind and Wegelius (r953a) have been able to demonstrate a patent foramen ovale or atrial septal defect using the intravenous method. This may have been possible due to the presence of a bidirectional shunt or temporary reversal of the shunt caused by a rise in right atrial pressure following the injection. By injecting contrast medium into the left atrium through a catheter it is possible to provide the surgeon with valuable information about the size and site of an atrial septal defect (Bjork et al., 1954).

\section{Patent Ductus Arteriosus}

Angiography is rarely required for the diagnosis of this condition. Usually Thoracic Aortography is the method of choice because of the shunt from the aorta to the pulmonary artery. Following opacification of the aortic arch, contrast medium flows into the pulmonary artery, providing conclusive evidence of the shunt and the ductus itself may be clearly shown. Localized dilatation of the aorta at the site of the ductus is common (Jonsson et al., $195 \mathrm{Ib}$ ) but it is not a pathognomonic sign.

Angiocardiography may be helpful in those cases where the development of pulmonary hypertension has produced a reversal of the shunt. Opacification of the descending aorta is then visible following filling of the pulmonary artery and before the opacification of the left atrium, left ventricle and ascending aorta.

\section{Coarctation of the Aorta}

The routine pre-operative use of angiography is not necessary if aortic grafts are available but is indicated in all cases with atypical features. The presence of the latter is usually evident from the clinical findings and the plain radiographs.
Angiography is also sometimes required for diagnosis in infants.

The anatomy is best demonstrated by Thoracic Aortography in the lateral position but Angiocardiography which is simpler, can provide good visualization especially if the injection is made through a catheter with the tip in the right atrium (or pulmonary trunk). In typical cases, which form about 90 per cent. of the total number, the ascending aorta appears lengthened and dilated, while the transverse arch is smaller than normal. The innominate and left subclavian arteries are usually much enlarged since they provide the collateral circulation. The coarctation, located between the origins of the left subclavian artery and ligamentum arteriosum, is short and may be dislocated forwards and medially. The descending portion of the aortic arch proximal to it, forms a stump of variable length and calibre. The post stenotic segment, which is often dilated, shows delay in filling to a degree dependant upon the severity of the stenosis and the efficiency of the collateral circulation.

Occasionally the coarctation involves a long segment of the aorta or lies proximal to the origin of the left subclavian artery, involving this or other branches of the arch. Rarely the stenosis may be demonstrated in the descending thoracic or abdominal aorta. Sometimes a patent ductuse arteriosus may be shown or rarely an anomalous right subclavian artery originating below the coarctation. Aneurysms arising from the aorta or intercostal arteries adjacent to the coarctation may also be demonstrated.

\section{Anomalous Pulmonary Venous Drainage}

Cases may be divided into two groups: I, Partial anomalous drainage; 2, Total anomalous drainage. The former group are usually asymptomatic and the diagnosis is often made on routine radiography. The anomalous vein is more common on the right and usually drains into the right atrium or the superior or inferior vena cava. On the left side it may enter the coronary sinus, a persistent left superior vena cava or the left innominate vein. Many cases of total anomalous drainage die in infancy as the presence of an atrial septal defect is necessary for survival. When the anomalous veins drain into a persistent left superior vena cava, the appearances on plain radiography are usually typical. Rarely all the veins enter the right atrium, coronary sinus or portal vein.

The angiocardiographic findings have been described by Snellen and Albers (1952). In group I, an area of decreased density may be produced in the contrast medium filling the superior vena cava or right atrium due to entry of 
blood from the anomalous vein. The latter opacifies with the normal pulmonary veins. In cases of group 2, with drainage into a persistent left superior vena cava, the right superior vena cava, right atrium and ventricle and pulmonary trunk are enlarged due to the increased venous return. There is successive filling of pulmonary arteries, pulmonary veins, left superior vena cava and left innominate vein with refilling of the right superior vena cava. The aorta may opacify early due to a shunt of contrast medium through the atrial septal defect.

\section{Other Congenital Anomalies}

Angiography has been used in conditions such as: Ebstein's disease, mitral atresia, idiopathic dilatation of the pulmonary artery, aortic and subaortic stenosis, aortico-pulmonary septal defect, congenital anomalies of the aortic arch and its branches and pulmonary arteriovenous aneurysms.

\section{Acquired Heart Disease (including the great vessels)}

Aortic Aneurysm. Angiography is of great value in the differentiation of aneurysms from mediastinal tumours. Its pre-operative use is advisable to demonstrate the anatomy. Aortography or angiocardiography may be employed, the L.A.O. position being the most informative. The origin and width of the neck of a saccular aneurysm, may be shown. Failure of the aneurysmal lumen to fill due to occlusion by thrombus is rare, but its opacification may be poor if the neck is very narrow. In addition, changes due to syphilitic aortitis may be visible, namely elongation and tortuosity of the aorta and irregular dilatation of its lumen, especially in the ascending aorta. Aneurysms may be multiple, and may extend into the brachiocephatic vessels. Displacement or obstruction of the superior vena cava or pulmonary vessels may be seen.

In dissecting aneurysms, an irregular narrowing of the lumen with diffuse thickening of the wall may be visible. Occasionally contrast medium may also enter the false lumen. Aneurysms of the sinuses of Valsalva, congenital or acquired, may show as a filling defect in the right atrium which later opacifies with the aorta.

\section{Mitral Valve Disease}

In this condition, delay in emptying and varying degrees of enlargement of the right atrium and ventricle, the pulmonary trunk and its primary divisions, and the left atrium can be shown by angiocardiography. Steiner and Goodwin (1954) observed in those cases with active pulmonary hypertension, a sudden diminution in calibre with increased tortuosity of the smaller pulmonary arteries similar to that seen in primary pulmonary hypertension.

Zinsser and Johnson (1953) used angiocardiography in the selection of patients for surgery. In mitral stenosis, they observed prolonged and dense opacification of the large left atrium in contrast to the poor opacification of the small left ventricle. In mitral incompetence, however, many cases showed no such difference in opacification and the left ventricle was enlarged. Bjork et al. (1955), have obtained angiocardiograms by injecting contrast medium into the left atrium following percutaneous puncture of this chamber through the posterior chest wall. In cases with incompetence they were able to show during ventricular systole, the defective closure of the mitral valve and also a regurgitant stream of contrast medium from the left atrium into the pulmonary veins. The method is not recommended for routine use due to the frequency of reactions.

Rarely, angiocardiography is of value by demonstrating thrombi or a myxoma in the left atrium which may simulate mitral stenosis.

\section{Pericardial Effusion}

Angiocardiography may be necessary to differentiate between a pericardial effusion, cardiac enlargement and rarely a cardiac tumor. Thisis most easily achieved by a study of the right atrium. In cases of pericardial effusion there is a wide gap between the atrial cavity and the pericardium due to fluid, whereas in cardiac enlargement the right atrial wall is less than $5 \mathrm{~mm}$. thick. In the rare cases of cardiac tumour an irregular thickening of the wall and sometimes a filling defect in the cardiac chamber may be seen. These findings are best demonstrated by injecting the contrast medium through a catheter with the tip pointing laterally in the right atrium.

\section{Constrictive Pericarditis}

Angiocardiography is occasionally of use when the diagnosis is in doubt and in localizing areas of thickened pericardium. The usual findings are enlargement of the superior and inferior vena cava but no evidence of constriction at their junction with the right atrium. Prolonged opacification of the cardiac chambers is due to the diminished cardiac output.

\section{BIBLIOGRAPHY}

ABRAHAMS, D. G., and WOOD, P. (195I), Brit. Heart f., 13, 519. BJORK, V. O. CRAFOORD, C., JOHNSSON, B., KJELLBERG, S. R., and RUDHE, U. (I954), Acta chir. Scand., 107, 499. BJORK, V. O., KJELLBERG, S. R., MALMSTROM, G., and RUUDHE, UU. (1955), Amer. Heart $\mathcal{F}$., 49, 719 .

BURFORD, T. H., and CARSON, M. J. (1948), F. Pediat., 33, 675. Continued on page 68 . 
aortic stenosis whether the origin of the second sound is aortic, pulmonary or both, but in any event, it may be well heard even when the stenosis is undoubtedly severe. Neither is the pulse a good guide as it may be in adults, for when the rate is rapid, the interval between beats is too short to allow recognition of a distinctive pattern. The radiogram may show a rounded ventricular shadow or left ventricular hypertrophy, but more often, and especially in young children, it is of little diagnostic value.
Acknowledgments

In compiling these facts, the writer has drawn freely from the literature and from the teaching and experience of many. $\mathrm{He}$ is particularly indebted to Dr. Paul Wood who initiated him in the physical signs of congenital heart disease and to Dr. Evan Bedford, his senior colleague at the Middlesex Hospital.

\section{BIBLIOGRAPHY}

WOOD, P. (1950), Brit. med. F., ii, 639. WOOD, P., MAGIDSON, O., and WILSON, P. A. O. (1954),
Brit. Heart Ғ., 16, 387.

\section{RHEUMATIC DISORDERS}

(Postgraduate Medical Journal)

Price 3s. 10d. post free

INTRODUCTION: THE RHEUMATIC DISEASES

F. Dudley Hart, M.D., F.R.C.P.

RECENT ADVANCES IN THE PATHOLOGY OF CHRONIC ARTHRITIS AND RHEUMATIC DISORDERS

D. H. Collins, O.B.E., M.D., M.R.C.P.

\section{RHEUMATOID ARTHRITIS}

J. J. R. Duthie, F.R.C.P.E.

\section{OSTEOARTHRITIS}

G. C. Lloyd-Roberts, M.B., F.R.C.S.
GOUT

R. M. Mason, D.M., M.R.C.P.

THE RARER ARTHROPATHIES

F. Dudley Hart, M.D., F.R.C.P.

PHYSICAL METHODS IN THE TREATMENT OF RHEUMATIC DISORDERS

W. S. Tegner, F.R.C.P.

THE SURGERY OF RHEUMATIC DISEASE

JohN Bastow, M.D., F.R.C.S.

Published by

THE FELLOWSHIP OF POSTGRADUATE MEDICINE 60, Portland Place, London, W.1

Bibliography continued from page 61-F. Norman Pattinson, M.B., B.Chir., D.M.R.D., F.F.R.

CASTEllanOS, A., and PEREIRAS, R. (1939), Rer. cubana Cardiol., 2, 187.

CAS'TELlanOS, A., PEREIRAS, R., and GARCIA, O. (I950), Amer. F. Roentgenol., 64, 255.

COPE, D. H. P. (1953), Brit. F. Anaesth., 25, 212.

DOTTER, C. T., and JACKSON, F. S. (I950), Radiology, 54, 527.

DOTTER, C. 'T., and STEINBERG, I. (I95I), 'Angiocardiography. Annals of Roentgenology,' voi. 20, New York, Paul B. Hoeber, Inc.

DOTTER, C. T., WETCHLER, M. S., and STEINBERG; I. (1953), Radiology, 60, 69I.

EMANUEL, R. W., and PAT'TINSON, J. N. (1955), Brit. Heart $\mathcal{F}$., in press.

JANKER, R. (1950), Fortschr. Rontgenstr., 72, 513.

JANKER, R., and HALLERBACH, H. (1951), Ibid., 75, 393.

JONSSON, G., BRODEN, B., and KARNELL, J. (1949), Acta radiol., Stockh., 32, 486 .

JONSSON, G. (1951 a), F. fac. Radiol., Lond., 3, 125.
JONSSON, G., BRODEN, B., and KARNELL, J. (r95 Ib), Acta radiol., Stockh., Suppl., 89.

JONSSON, G., BRODEN, B., and KARNELL, J. (1953), Ibid., 40, 547 .

LIND, J., and WEGELIUS, C. (1949), $\mathcal{F}$. fac. Radiol., Lond., I, 87.

LIND, J., and WEGELIUS, C. (1953a), Circulation, 7, 819.

NUVOLI, I. (1936), Policlinico, 43, 227.

SNELLEN, H. A., and ALBERS, F. H. (1952), Circulation, 6, Sor.

STEINER, R. E., and GOODWIN, J. F. (1954), F. fac. Radiol., Lond., 5 , 167.

SUTTON, D. (I955), F. fac. Radiol., Lond., in press.

WEGELIUS, C., and LIND, J. (r953b), Acta radiol., Stockh., 39, 177 .

WICKBOM, I. (1952), Ibid., 38, 343, 350.

ZINSSER, H. F., and JOHNSON, J. (1953), Ann. int. Med., 39, 1200. 